\title{
Plastic potential: how the phenotypes and adaptations of pathogens are influenced by microbial interactions within plants Kayleigh R O'Keeffe ${ }^{1}$, Ignazio Carbone ${ }^{2}$, Corbin D Jones ${ }^{1,3}$ and
Charles E Mitchell
}

Predicting the effects of plant-associated microbes on emergence, spread, and evolution of plant pathogens demands an understanding of how pathogens respond to these microbes at two levels of biological organization: that of an individual pathogen and that of a pathogen population across multiple individual plants. We first examine the plastic responses of individual plant pathogens to microbes within a shared host, as seen through changes in pathogen growth and multiplication. We then explore the limited understanding of how within-plant microbial interactions affect pathogen populations and discuss the need to incorporate populationlevel observations with population genomic techniques. Finally, we suggest that integrating across levels will further our understanding of the ecological and evolutionary impacts of within-plant microbial interactions on pathogens.

\footnotetext{
Addresses

${ }^{1}$ Department of Biology, University of North Carolina at Chapel Hill, Chapel Hill, NC, USA

${ }^{2}$ Center for Integrated Fungal Research, Department of Entomology and Plant Pathology, North Carolina State University, Raleigh, NC, USA

${ }^{3}$ Integrative Program for Biological \& Genome Sciences, University of North Carolina at Chapel Hill, Chapel Hill, NC, USA

${ }^{4}$ Curriculum for the Environment and Ecology, University of North Carolina, Chapel Hill, NC, USA
}

Corresponding author: O’Keeffe, Kayleigh R (kokeeffe@live.unc.edu)

\section{Current Opinion in Plant Biology 2017, 38:78-83}

This review comes from a themed issue on Biotic interactions

Edited by Silke Robatzek and Sarah Lebeis

For a complete overview see the Issue and the Editorial

Available online 12th May 2017

http://dx.doi.org/10.1016/j.pbi.2017.04.014

\section{Introduction}

Plants are host to a diverse set of mutualistic, commensal, and parasitic microbes, referred to collectively as the phytobiome. The microbes (bacteria, fungi, and viruses) of the phytobiome live in, on and around their plant hosts. Historically difficult to interrogate - largely because work was limited to identifying microbial species by symptoms or by using culture-based methods - the composition, dynamics, and functional biology of the phytobiome are now being revealed by next-generation sequencing technologies [1].

Within the phytobiome, plant pathogens are among the best-studied microbes due to their negative impacts on their hosts. Infectious disease has historically been studied in a one host-one pathogen framework, but the field thanks in part to next-generation sequencing [2] - is now appreciating the role and complexity of within-host microbial interactions. Within a shared host, pathogens may interact by directly facilitating or interfering with each other, or indirectly, mainly via host resources or the host's immune system [3]. Different plant pathogens may stimulate different signaling pathways, and the cross talk between such pathways can affect overall disease. Additionally, plants can attain bacteria-induced systemic acquired resistance (SAR), induced systemic resistance (ISR) or induced systemic susceptibility (ISS), in which prior infection or treatment results in increased resistance or susceptibility to subsequent invasions by pathogens [4,5]. Pathogens may also interact with non-parasitic microbes within the plant [6,7].

A key question in infection biology is how do these interactions shape pathogens in both the near and long term? Do the plastic responses of individual pathogens affect pathogen population genetic structure, altering their adaptability? Phenotypic plasticity is the ability of one genotype to express different phenotypes in different environmental contexts-an ability that is under genetic control and heritable [8]. For example, fungal pathogens such as Mycosphaerella graminicola and Rhynchosporium commune have been shown to exhibit phenotypic plasticity in growth rate and response to different temperatures [52,53]. Evolutionary theory juxtaposes genetic responses against plastic responses; however, there is conflict among both theoretical and empirical studies as to the direction of the connection between adaptive plasticity and evolution [9].

Studies that offer insight into a system on multiple levels of biological organization can help resolve this tension. Here we consider how microbial interactions shape plant pathogen growth and multiplication within individual plants and subsequent transmission between plants. 
We emphasize that an understanding of plastic and genetic responses is critical at two key levels: the pathogen individual and the pathogen population. With a focus on fungal pathogens, we examine what we have learned about within-plant microbial interactions and disease from each of these levels while stressing the plastic responses of individual pathogens and genetic responses of pathogen populations to microbial interactions. Finally, we emphasize the novel insights that can be gained by integrating studies across levels.

\section{Individual-level plasticity}

Microbes are known to be major drivers of shifts in plant phenotype [10]. As noted above, pathogens induce complex defensive signaling pathways in plants [11,12], and beneficial microbes such as arbuscular mycorrhizal fungi facilitate increased nutrient uptake in plants $\left[13^{\circ}\right]$, which in turn alters the plant's physiological components. Less attention has been given to the plastic responses of plant pathogens to other components of the phytobiome, and how those responses, in turn, affect the infection process and consequent disease progression.

The best data on responses of pathogens to microbial interactions is from agriculturally important systems. This work suggests that specific interactions can alter the phenotypes of pathogens. For example, one fungus infecting maize, Fusarium verticillioides, modulates the growth of a maize fungal pathogen, Ustilago maydis, consequently decreasing the aggressiveness of $U$. maydis towards its host [14]. Transcriptomic analyses of $F$. verticillioides and $U$. maydis grown together in liquid culture suggests that $U$. maydis responds to $F$. verticillioides through the overexpression of siderophore biosynthetic genes and genes involved in toxin synthesis [15]. The interaction also results in the shutdown of antifungal metabolites like ustilagic acid in $U$. maydis. The phenotypic response of $U$. maydis to $F$. verticillioides is, therefore, genetically controlled, although its heritability has yet to be estimated. These findings suggest that $U$. maydis responds plastically to coinfection with $F$. verticillioides.

Interactions also occur indirectly between coinfecting pathogens through the defense pathways of the plant host. Plants respond to enemies with different infection strategies through different defense pathways, and cross talk between these pathways when two different pathogens infect a plant simultaneously can result in antagonism or synergism between the pathogens [16]. Necrotrophic pathogens, which kill host cells to feed and reproduce, induce defenses mediated by jasmonic acid. Biotrophic pathogens, which feed and reproduce on live host cells, induce defenses mediated by salicylic acid. Spoel et al., [17] showed that salicylic acid-mediated defenses induced by the bacterium Pseudomonas syringae made Arabidopsis thaliana more susceptible to infection by the necrotrophic fungal pathogen Alternaria brassicola by suppressing the jasmonic acid signaling pathway. Disease symptoms from $A$. brassicola progressed more quickly, and A. brassicola produced more spores when the salicylic acid pathway suppressed the jasmonic acid pathway. Plastic responses to microbial interactions can therefore occur through indirect means. Although genes expressed by $A$. brassicola during infection of $A$. thaliana have been characterized [18], the genes that specifically respond to the presence/absence of jasmonic acid still need to be characterized to determine if this phenotypic response is genetically controlled [19].

To date, studies investigating the role of within-plant microbial interactions on plant pathogens primarily focus on phenotypic effects. In order for plasticity to be fully understood, the underlying genetic mechanisms controlling these differences need to be considered. As seen with the Ustilago-Fusarium example, gene expression studies are a powerful tool for assessing if phenotypic changes in response to biotic or abiotic factors are genetically controlled and give insight into what the controlling genetic mechanisms are. Characterizing gene expression in in vitro confrontation assays, though informative, can miss interactions that are mediated by the host. AdameÁlvarez et al. [7], for instance, investigated the effect asymptomatic endophytic fungal strains had on pathogens both in vitro and in planta. While in vitro confrontation assays between endophytes and three pathogens consistently showed symmetric reciprocal effects, in planta experiments revealed that these effects varied depending on the order of the arrival of the endophyte and pathogen on their shared host. One solution to the challenges of characterizing molecular mechanisms of plastic responses is to simultaneously characterize gene expression of both organisms in situ. Dual RNA-seq, sequencing host and pathogen transcriptomes in parallel, has the potential to meet this need [20] and can be applied to the frequent cases of host tissue infected with more than one microbe.

\section{Pathogen population}

In contrast to near-term plastic responses in pathogen individuals, long-term changes within pathogen populations are thought to demand evolved responses. The evolutionary forces shaping plant pathogen populations can determine a pathogen's evolutionary potential and predict its ability to shift hosts, expand its range, and emerge as a disease-causing agent $[21,22]$. Within-host microbial interactions may impact the evolutionary dynamics of pathogen populations, but few population genetic studies have focused on how interspecific microbial interactions affect the evolution of plant pathogens. Application of population genetic techniques to plant pathogen populations can address other questions related to the evolutionary and demographic processes shaping population structure. For example, these techniques can elucidate the processes driving or constraining the 
divergence of closely related taxa [23]. A study of wheat blast disease, caused by species within the fungal genus Pyricularia, compared strains of these different species isolated from wheat and rice fields across Brazil. They found that contrary to the previously held thought that wheat blast disease emerged through a host jump from strains infecting rice, the pathogen likely emerged through a host jump from a population infecting wild grasses $[23,24]$. Further work on this fungal genus determined that Pyricularia oryzae isolates from ryegrass show evidence of recent evolutionary events, and that gene-forgene interactions resulted in resistance of common wheat to these isolates of Pyricularia oryzae [25]. These techniques could also be applied to findings like that of Gold et al. [26], who observed competitive exclusion between species of Microbotryum fungi during infection of Silene vulgaris. These observations are consistent with previous findings of a positive correlation between genetic distance and antagonistic interaction among Microbotryum, and this segregation among host plants may contribute to barriers to gene flow between species [27]. Badouin et al., [28] further identified putative candidate effector genes from genomic regions that harbor signatures of selective sweeps in Microbotryum lychnidis-dioicae and M. silenesdioicae, causing disease on S. latifolia and S. dioica, respectively. How a pathogen is able to expand its host range has been of great interest as emerging infectious diseases increase, with a new focus on the role of pan-genomes in pathogen emergence and adaptation. A pan-genome refers to the set of all possible genomic sequences that can belong to an organism or organisms within a predefined phylogenetic clade. Comparative population genomic studies have revealed the existence of intraspecific pan-genomes in bacteria and fungi that may contribute to niche specialization, pathogenicity and host specificity [29,30]. Complementing studies like those of Microbotryum species with hypothesis-driven population genomic approaches applied to closely related species could provide clear evidence of the evolutionary history and potential of a system as it relates to within-host microbial interactions.

Measures of genetic structure and diversity of plant pathogen populations are also commonly used to investigate their evolutionary history and their potential to generate new variation in traits that influence interactions with their hosts [23,31,32]. For example, Walker et al., $\left[33^{\circ}\right]$ investigated population subdivision in the multihost fungal pathogen, Botrytis cinerea, and found a weak association between population structure and geography but a clear differentiation by the host plant of origin. We believe extending these investigations of population structure to populations with known within-host microbial interactions could determine if there is an association between population subdivision and presence/absence of other microbes. One could, for instance, sequence individuals of a given plant pathogen species from hosts that are or are not coinfected with another pathogen. This would elucidate the effects these interactions may have on the evolutionary dynamics of pathogens.

Genome-wide association studies (GWASs) of plant pathogens aim to determine the genes responsible for specific phenotypes, including those potentially modulated by the microbial community. Talas and McDonald [34] quantified intraspecific variation in traits like aggressiveness in isolates of Fusarium graminearum, a common fungal pathogen of wheat, barley, and maize. They used RAD-seq to conduct a GWAS of these isolates. The study identified a gene encoding a RAS GTPase-activating protein as an important contributor to aggressiveness (reviewed in Ref. [23]). Pańka et al. [35] characterized intraspecific variation in the suppressive effect of the tall fescue endophyte Epichloë coenophiala on the pathogen Rhizoctonia zeae. The suppressive effect of the endophyte on pathogen infectivity and growth ranged from $30 \%$ to $52 \%$ (a significant parasite isolate $\mathrm{x}$ endophyte interaction), indicating standing genetic variation for parasite traits conferring partial resistance to, or tolerance of, the suppressive effects of the endophyte. More studies need to incorporate measures of intraspecific variation in the effects of within-host microbial interactions on pathogens. Complementing such studies with GWASs would allow the genes contributing to the responses of pathogens to these microbes to be identified.

Recent work suggests that not all plasticity is adaptive and that selection could be functioning to increase the degree of plasticity and the maintenance of genetic mechanisms that promote diversity. For example, despite any known function of secondary metabolite aflatoxin in pathogenesis and microbial competition, recombination hotspots have been shown to associate with qualitative and quantitative differences in aflatoxin concentrations in Aspergillus [36,37], lineage-specific gene loss has occurred in the aflatoxin gene cluster in A. flavus [38], and balancing selection has maintained chemotype-specific lineages in A. parasiticus [39]. Aflatoxin has no known function in pathogenesis or microbial competition, but may have necessary, nonspecific functions in nature, such as niche retention and defense against opportunistic pathogens [40]. Secondary metabolites can often be involved in interactions between microbes, and conversely, microbial interactions can also affect the production of secondary metabolites [41]. Performing studies like those used to characterize the genetic patterns underlying aflatoxin production to investigate other secondary metabolites involved in microbial interactions would facilitate a better understanding of related evolutionary dynamics.

\section{Integrating across levels}

Population genetic processes can drive shifts in plasticity, and plasticity can facilitate or constrain the population genetic process of adaptation. Often investigated in 
isolation, these two evolutionary forces inherently cooccur within a population of pathogens. To date, it remains unclear how the connection between these two evolutionary forces shapes pathogen-phytobiome interactions. The only field experiment testing the connection between plasticity and adaptation $\left[42^{\circ \bullet}\right]$, conducted using Trinidadian guppies (Poecilia reticulata), supports models predicting that adaptive plasticity constrains evolution [43] and that non-adaptive plasticity potentiates evolution by increasing the strength of directional selection [44].

Standard theory for evolution of virulence in pathogens has only recently started to incorporate phenotypic plasticity [45-47] even though both the evolution and phenotypic plasticity of virulence requires genetic control. Phytobiome experiments that parallel the work in $P$. reticulata could provide a means of exploring this connection. Such studies could involve reciprocally transplanting isolates of a pathogen between areas with and without a potentially interacting microbe. Testing for evolutionary divergence in gene expression patterns in transplanted individuals would allow for the direction of plasticity in gene expression and the direction of adaptive evolution to be compared. Plasticity in gene expression would measure differences in transcription between genetically identical isolates inoculated in areas with and without a potentially interacting microbe, while differences between populations measured under the same conditions for multiple generations represent heritable differences. Furthermore, integrating transcriptomic data with population genetic data using coalescent models would facilitate the characterization of relationships among differential gene expression, population history, and ecological process [48]. More specifically, such integration would allow researchers to address questions like if lineages with high plasticity in gene expression harbor more genetic variation and if plastic responses

Figure 1

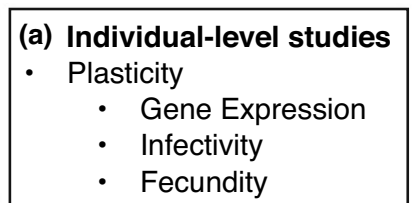

(b) Population-level studies

- Transmission

- Population Structure

- Population Genetic Processes

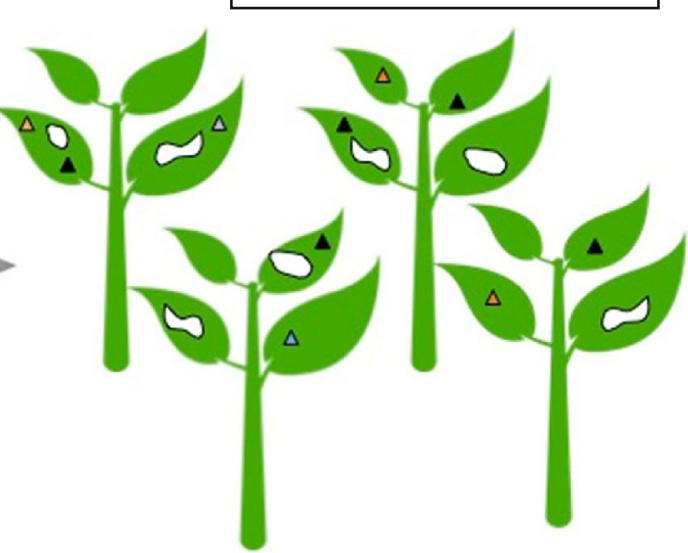

(c) Integrated studies

- Do plastic responses in gene expression occur at loci known to undergo balancing selection?

- Do parasite lineages with high plasticity in gene expression also harbor more genetic variation? In those lineages, do we see evidence of a recent population bottleneck and expansion as expected under a selective sweep?

- Do plastic responses in gene expression occur at loci known to undergo balancing selection?

Current Opinion in Plant Biology 
in gene expression occur at loci known to undergo balancing selection (Figure 1).

For these cross-level questions, foliar fungal pathogens offer a tractable system. As evidenced in this review, foliar fungal pathogens exhibit plastic responses to within-host microbial interactions, and these interactions can have important effects on population dynamics. For most foliar fungal pathogens, individuals can be visually distinguished, allowing for individual-level and population-level studies to be readily integrated, and are able to be manipulated experimentally $\left[49^{\bullet \bullet}, 50\right]$. Finally, fungi are an important component of the microbiome within leaves, making them a useful model for microbiome biology $\left[51^{\circ}\right]$.

\section{Conclusions}

We suggest that the microbial community surrounding a plant pathogen shapes both that pathogen's immediate phenotype and its evolutionary potential. Ultimately, proof of this idea requires interdisciplinary approaches spanning ecology, evolutionary biology and plant pathology that integrate individual-level and population-level studies. To date, most studies of the effects of withinplant microbial interactions are at the individual level, focusing on the plastic responses of individual parasites to coinfecting microbes. Studies of population-level responses to microbial interactions are currently limited; conducting more such studies would increase understanding of how microbial interactions affect evolutionary responses, which in turn will give new insight into how virulent plant pathogens arise. Furthermore, integrating individual-level and population-level studies of the same system would provide much needed insight on the connection between phenotypic plasticity and evolution. Such integration would be invaluable when considering short-term and long-term results of disease management efforts related to emerging diseases and pathogens expanding their host range. Foliar fungal pathogens offer a tractable system to apply such a paradigm. With more population-level studies looking at the evolutionary effects of within-host microbial interactions, and the integration of this population-level data with data from the individual level, we will be able to improve ecological and evolutionary understanding of plant pathogens.

\section{Acknowledgements}

This work was supported by the NSF-USDA joint program in Ecology and Evolution of Infectious diseases (USDA-NIFA AFRI grant 2016-6701325762) to C.E.M., C.D.J., and I.C., the Agriculture and Food Research Initiative Competitive Grants Program (USDA-NIFA AFRI grant 201368004-20359) to I.C., the National Science Foundation to C.D. J. (DEB1457690) and I.C. (DEB-1541418), and an NSF graduate research fellowship to K.R.O.

\section{References and recommended reading}

Papers of particular interest, published within the period of review, have been highlighted as:

- of special interest

-. of outstanding interest
1. Lundberg DS, Lebeis SL, Paredes SH, Yourstone S, Gehring J, Malfatti S, Tremblay J, Engelbrektson A, Kunin V, del Rio TG et al.: Defining the core Arabidopsis thaliana root microbiome. Nature 2012, 488:86-90.

2. Müller DB, Vogel C, Bai Y, Vorholt JA: The plant microbiota: systems-level insights and perspectives. Annu. Rev. Genet. 2016, 50:211-234.

3. Alizon S, de Roode JC, Michalakis Y: Multiple infections and the evolution of virulence. Ecol. Lett. 2013, 16:556-567.

4. $F u Z Q$, Dong $X$ : Systemic acquired resistance: turning local infection into global defense. Annu. Rev. Plant Biol. 2013, 64:839-863.

5. Pieterse CMJ, Zamioudis C, Berendsen RL, Weller DM, Van Wees SCM, Bakker PAHM: Induced systemic resistance by beneficial microbes. Annu. Rev. Phytopathol. 2014, 52:347-375.

6. May G, Nelson P: Defensive mutualisms: do microbial interactions within hosts drive the evolution of defensive traits? Funct. Ecol. 2014, 28:356-363.

7. Adame-Álvarez R-M, Mendiola-Soto J, Heil M: Order of arrival shifts endophyte-pathogen interactions in bean from resistance induction to disease facilitation. FEMS Microbiol. Lett. 2014, 355:100-107.

8. Pigliucci M: Phenotypic Plasticity: Beyond Nature and Nurture. Johns Hopkins University Press; 2001.

9. Levis NA, Pfennig DW: Evaluating plasticity-first evolution in nature: key criteria and empirical approaches. Trends Ecol. Evol. 2016, 31:563-574.

10. Friesen ML, Porter SS, Stark SC, von Wettberg EJ, Sachs JL, Martinez-Romero E: Microbially mediated plant functional traits. Annu. Rev. Ecol. Evol. Syst. 2011, 42:23-46.

11. Derksen H, Rampitsch C, Daayf F: Signaling cross-talk in plant disease resistance. Plant Sci. 2013, 207:79-87.

12. Jones JDG, Vance RE, Dangl JL: Intracellular innate immune surveillance devices in plants and animals. Science 2016, 354.

13. Rúa MA, Antoninka A, Antunes PM, Chaudhary VB, Gehring C,

- Lamit LJ, Piculell BJ, Bever JD, Zabinski C, Meadow JF et al: Home-field advantage? evidence of local adaptation among plants, soil, and arbuscular mycorrhizal fungi through metaanalysis. BMC Evol. Biol. 2016, 16:122.

Using meta-analysis, the authors illustrated that geographic origin of plants relative to the origin of AM fungal communities and soil is an important determinant of the effect of mycorrhizal inoculation on plant growth. They demonstrate the importance of considering local adaption in mycorrhizal symbiosis.

14. Rodriguez Estrada AE, Jonkers W, Corby Kistler H, May G: Interactions between Fusarium verticillioides, Ustilago maydis, and Zea mays: an endophyte, a pathogen, and their shared plant host. Fungal Genet. Biol. 2012, 49:578-587.

15. Jonkers W, Rodriguez Estrada AE, Lee K, Breakspear A, May G Kistler HC: Metabolome and transcriptome of the interaction between Ustilago maydis and Fusarium verticillioides in vitro. Appl. Environ. Microbiol. 2012, 78:3656-3667.

16. Koornneef $A$, Pieterse $\mathrm{CMJ}$ : Cross talk in defense signaling Plant Physiol. 2008, 146:839-844.

17. Spoel SH, Johnson JS, Dong X: Regulation of tradeoffs between plant defenses against pathogens with different lifestyles. Proc. Natl. Acad. Sci. U. S. A. 2007, 104:18842-18847.

18. Cramer RA, Lawrence CB: Identification of Alternaria brassicicola genes expressed in planta during pathogenesis of Arabidopsis thaliana. Fungal Genet. Biol. 2004, 41:115-128.

19. Cho Y: How the necrotrophic fungus Alternaria brassicicola kills plant cells remains an enigma. Eukaryot. Cell 2015, 14: 335-344

20. Westermann AJ, Gorski SA, Vogel J: Dual RNA-seq of pathogen and host. Nat. Rev. Microbiol. 2012, 10. 
21. Parker IM, Gilbert GS: The evolutionary ecology of novel plantpathogen interactions. Annu. Rev. Ecol. Evol. Syst. 2004, 35: 675-700.

22. Gladieux P, Feurtey A, Hood ME, Snirc A, Clavel J, Dutech C, Roy M, Giraud T: The population biology of fungal invasions. Mol. Ecol. 2015, 24:1969-1986.

23. Grünwald NJ, McDonald BA, Milgroom MG: Population genomics of fungal and oomycete pathogens. Annu. Rev. Phytopathol. 2016, 54:323-346.

24. V. Castroagudin L, Moreira SI, Pereira DAS, Moreira SS, Brunner PC, Maciel JLN, Crous PW, McDonald B, Alves E, Ceresini PC: Pyricularia graminis-tritici, a new Pyricularia species causing wheat blast. Persoonia 2016, 37:199-216 http:// dx.doi.org/10.3767/003158516X692149.

25. Tosa $Y$, Inoue $Y$, Trinh TPV, Chuma I: Genetic and molecular analyses of the incompatibility between Lolium isolates of Pyricularia oryzae and wheat. Physiol. Mol. Plant Pathol. 2016, 95:84-86.

26. Gold A, Giraud T, Hood ME: Within-host competitive exclusion among species of the anther smut pathogen. BMC Ecol. 2009, 9:11.

27. Koskella B, Giraud T, Hood ME: Pathogen relatedness affects the prevalence of within-host competition. Am. Nat. 2006, 168:121-126.

28. Badouin H, Gladieux P, Gouzy J, Siguenza S, Aguileta G, Snirc A Le Prieur S, Jeziorski C, Branca A, Giraud T: Widespread selective sweeps throughout the genome of model plant pathogenic fungi and identification of effector candidates. Mol. Ecol. 2017, 26:2041-2062 http://dx.doi.org/10.1111/ mec.13976.

29. Thynne E, McDonald MC, Solomon PS: Phytopathogen emergence in the genomics era. Trends Plant Sci. 2015, 20: $246-255$

30. Xu Y, Zhu Y, Wang Y, Chang Y-F, Zhang Y, Jiang X, Zhuang X, Zhu $Y$, Zhang J, Zeng $L$ et al.: Whole genome sequencing revealed host adaptation-focused genomic plasticity of pathogenic Leptospira. Sci. Rep. 2016, 6:20020.

31. Grünwald NJ, Goss EM: Evolution and population genetics of exotic and re-emerging pathogens: novel tools and approaches. Annu. Rev. Phytopathol. 2011, 49:249-267.

32. Linde CC: Population genetic analyses of plant pathogens: new challenges and opportunities. Australas. Plant Pathol. 2010, 39:23-28.

33. Walker A-S, Gladieux P, Decognet V, Fermaud M, Confais J,

- Roudet J, Bardin M, Bout A, Nicot CP, Poncet C et al.: Population structure and temporal maintenance of the multihost fungal pathogen Botrytis cinerea: causes and implications for disease management. Environ. Microbiol. 2015, 17:1261-1274.

Using microsatellite markers, the authors show that there is a strong association between population subdivision and host plant of origin for a plant pathogen, Botrytis cinerea.

34. Talas F, McDonald BA: Genome-wide analysis of Fusarium graminearum field populations reveals hotspots of recombination. BMC Genomics 2015, 16:996.

35. Pańka D, West CP, Guerber CA, Richardson MD: Susceptibility of tall fescue to Rhizoctonia zeae infection as affected by endophyte symbiosis. Ann. Appl. Biol. 2013, 163:257-268.

36. Moore GG, Elliott JL, Singh R, Horn BW, Dorner JW, Stone EA, Chulze SN, Barros GG, Naik MK, Wright GC et al: Sexuality generates diversity in the aflatoxin gene cluster: evidence on a global scale. PLoS Pathog. 2013, 9:e1003574.

37. Olarte RA, Worthington CJ, Horn BW, Moore GG, Singh R, Monacell JT, Dorner JW, Stone EA, Xie D-Y, Carbone I: Enhanced diversity and aflatoxigenicity in interspecific hybrids of Aspergillus flavus and Aspergillus parasiticus. Mol. Ecol. 2015, 24:1889-1909.
38. Moore GG, Singh R, Horn BW, Carbone I: Recombination and lineage-specific gene loss in the aflatoxin gene cluster of Aspergillus flavus. Mol. Ecol. 2009, 18:4870-4887.

39. Carbone I, Jakobek JL, Ramirez-Prado JH, Horn BW: Recombination, balancing selection and adaptive evolution in the aflatoxin gene cluster of Aspergillus parasiticus. Mol. Ecol. 2007, 16:4401-4417.

40. Carbone RC: Form and function in the evolution of dermatophytes. In Biology of Dermatophytes and other Keratinophilic Fungi. Edited by Kushwaha RKS, Guarro J. Revista Iberoamericana de Micología; 2000:30-43.

41. Netzker T, Fischer J, Weber J, Mattern DJ, König CC, Valiante V Schroeckh V, Brakhage AA: Microbial communication leading to the activation of silent fungal secondary metabolite gene clusters. Front. Microbiol. 2015, 6:299.

42. Ghalambor CK, Hoke KL, Ruell EW, Fischer EK, Reznick DN,

- Hughes KA: Non-adaptive plasticity potentiates rapid adaptive evolution of gene expression in nature. Nature 2015, 525: 372-375

Using transcriptomic sequencing, the authors provide evidence that adaptive plasticity constrains evolution. This is the first field test of the connection between phenotypic plasticity and evolution.

43. Paenke I, Sendhoff B, Kawecki TJ: Influence of plasticity and learning on evolution under directional selection. Am. Nat. 2007, 170:E47-E58

44. Conover DO, Duffy TA, Hice LA: The covariance between genetic and environmental influences across ecological gradients. Ann. N. Y. Acad. Sci. 2009, 1168:100-129.

45. Taylor PD, Day T, Nagy D, Wild G, André J-B, Gardner A: The evolutionary consequences of plasticity in host-pathogen interactions. Theor. Popul. Biol. 2006, 69:323-331.

46. Choisy M, de Roode JC: Mixed infections and the evolution of virulence: effects of resource competition, parasite plasticity, and impaired host immunity. Am. Nat. 2010, 175:E105-E118.

47. Brown SP, Cornforth DM, Mideo N: Evolution of virulence in opportunistic pathogens: generalism, plasticity, and control. Trends Microbiol. 2012, 20:336-342.

48. Nabholz B, Sarah G, Sabot F, Ruiz M, Adam H, Nidelet S, Ghesquière A, Santoni S, David J, Glémin S: Transcriptome population genomics reveals severe bottleneck and domestication cost in the African rice (Oryza glaberrima). Mol. Ecol. 2014, 23:2210-2227.

49. Ritpitakphong U, Falquet L, Vimoltust A, Berger A, Métraux J-P,

-• L'Haridon F: The microbiome of the leaf surface of Arabidopsis protects against a fungal pathogen. New Phytol. 2016 210:1033-1043.

The significant contribution of the phyllosphere microbiome to plant resistance in Arabidopsis mutants resistant to pathogen, Botrytis cinerea, was illustrated through experimental inoculations

50. Shehata HR, Lyons EM, Jordan KS, Raizada MN: Bacterial endophytes from wild and ancient maize are able to suppress the fungal pathogen Sclerotinia homoeocarpa. J. Appl. Microbiol. 2016, 120:756-769.

51. Christian N, Whitaker B, Clay K: Microbiomes: unifying animal - and plant systems through the lens of community ecology theory. Front. Microbiol. 2015, 6:869.

The review emphasizes the importance of unifying advances made in microbiome studies of animals and plants. arguing that community ecology offers a foundation to support such connections.

52. Zhan J, McDonald BA: Thermal adaptation in the fungal pathogen Mycosphaerella graminicola. Mol. Ecol. 2011, 20:1689-1701.

53. Stefansson TS, McDonald BA, Willi Y: Local adaptation and evolutionary potential along a temperature gradient in the fungal pathogen Rhynchosporium commune. Evol. Appl. 2013, 6:524-534. 